The experimental verification of the static magnetic interaction (a) was made by the $g$ determination of the $0.08 \mu \mathrm{sec}$ state of $\mathrm{Cd}^{111} .^{1}$ The presence of the static quadrupole interaction (b) was indicated by the correlation measurements on single crystals of In, ${ }^{2}$ and also by the results obtained with liquid sources. ${ }^{3,4}$

The influence of the randomly fluctuating interactions (c) and (d), such as must exist in liquids, on the angular correlation of nuclear radiations has been considered theoretically by Pound and Abragam, ${ }^{5,6}$ but no direct experimental evidence for this interaction type has been given so far.

If the directional correlation is expressed in the form $W(\theta)$ $=1+\Sigma_{k} G_{k} A_{k} P_{k}(\cos \theta)$, where $G_{k}=1$ corresponds to the undisturbed correlation, Abragam and Pound's model for the timedependent quadrupole interaction in a liquid yields the following expression for the attenuation factors $G_{k}:{ }^{6}$

$G_{k}=\frac{1}{1+\lambda_{k} \tau_{N}} \frac{1-e^{-\delta-\lambda_{k} \tau_{N} \delta}}{1-e^{-\delta}}$

$\lambda_{k}=\frac{3}{80}\left(\frac{e Q}{\hbar}\right)^{2}\left\langle\left(\frac{\partial^{2} V}{\partial z^{\prime 2}}\right)\right\rangle_{\mathrm{Av}} \cdot \tau_{c} \frac{k(k+1)[4 I(I+1)-k(k+1)-1]}{I^{2}(2 I-1)^{2}}$,

where $\delta=\tau_{0} / \tau_{N}$ is the ratio of the finite resolving time $\tau_{0}$ of the coincidence analyzer to the lifetime $\tau_{N}$ of the intermediate nuclear state of angular momentum $I . \tau_{c}$ is closely related to the characteristic time which, in Debye's theory of dielectric dispersion in polar liquids, characterizes the time interval during which molecular orientation persists. ${ }^{7} \tau_{c}$ is connected with the macroscopic viscosity $\eta$ of the liquid. A crude approximation for $\tau_{c}$ is $^{8}$

$$
\tau_{c}=4 \pi \eta a^{3} / 3 k T,
$$

where $a$ is the molecular radius. Hence $G_{k}$ depends on the viscosity $\eta$ of the liquid source.

The influence of the viscosity on the directional correlation $W(\theta)=1+A_{2} G_{2} P_{2}(\cos \theta)$ of the $\mathrm{Cd}^{111}$ gamma rays was investigated by measuring $A_{2} G_{2}$ with sources of very dilute aqueous $\mathrm{InCl}_{3}$ solution to which different amounts of glycerine were added (Fig. 1)

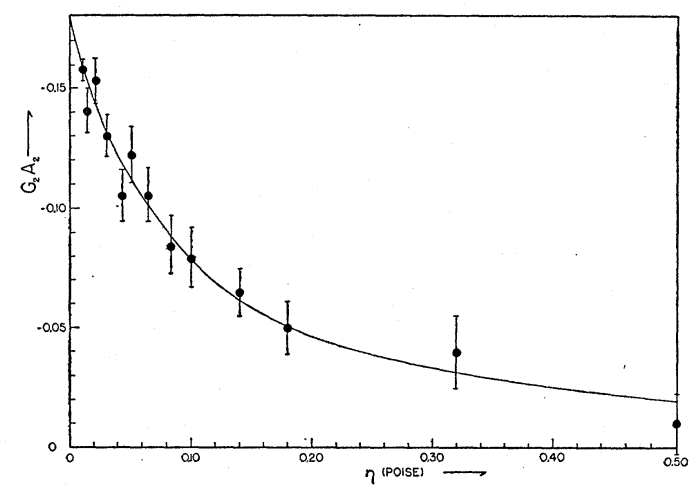

FIG. 1. Attenuation of the C $\mathrm{d}^{111}$ gamma-gamma directional correlation in water-glycerine mixtures of different viscosity $\eta$. The solid line represents the theoretical curve for $A_{2}=-0.18$ and $\lambda_{2}=25 \eta_{\text {poise. }}$

and also by changing the temperature of a 55 percent waterglycerine mixture. The resolving time of the coincidence analyzer was $0.125 \mu \mathrm{sec}(\delta=1.0)$.

That the observed attenuation is due mainly to the timedependent quadrupole interaction and not to magnetic relaxation phenomena is indicated by the fact that the addition of paramagnetic ions $\left(\mathrm{Fe}^{+++}\right)$to the liquid does not attenuate the correlation. ${ }^{3}$

Combining expressions (1) and (2), $A_{2} G_{2}$ can be expressed as a function of $\eta$, the coefficient $A_{2}$ for the unperturbed correlation and the quadrupole interaction term $e Q\left(\partial^{2} V / \partial z^{2}\right) / h$ being left as parameters. A least-squares fit of the data yields -0.18 for $A_{2}$ and $500 \mathrm{Mc} / \mathrm{sec}$ for $e Q\left(\partial^{2} V / \partial z^{2}\right) / h$, assuming $1.5 \times 10^{-8} \mathrm{~cm}$ for $a$.
The value of $A_{2}$ agrees with the value $A_{2}=-0.17 \pm 0.01$ corresponding to the maximum anisotropy found with the single, crystal sources. As to the electric quadrupole interaction energy the value obtained here should not be considered more than a rough estimate, due to the crude approximation for $\tau_{c}$ and also due to the fact that $\partial^{2} V / \partial z^{\prime 2}$ is probably not the same for the different water-glycerine mixtures. Nuclear magnetic resonance absorption experiments, however, show a remarkable constancy of the details of the molecular motion for different water-glycerine mixtures up to $\eta=1$ poise at least as far as the magnetic interaction is concerned.

Further experiments, to study the validity of the assumptions made above and to arrive at a better estimate of the quadrupole interaction, are in progress and will be reported later.

$$
\begin{aligned}
& \text { * Supported by the U. S. Atomic Energy Commission. } \\
& 1 \text { H. Aeppli et al., Helv. Phys. Acta 25, } 339 \text { (1952). }
\end{aligned}
$$

$1 \mathrm{H}$. Aeppli et al., Helv. Phys. Acta 25, 339 (1952).

2 Albers-Schonberg, Hänni, Heer, Novel, and Scherrer, Phys. Rev. 90, 322 (1953).

${ }_{3}$ R. M. Steffen, Phys. Rev. 90, 1119 (1953)

4 Albers-Schonberg, Heer, Novel, and Rüetschi, Phys. Rev. 91, 199 (1953).

5 R. V. Pound and A. Abragam, Phys. Rev. 90, 993 (1953).

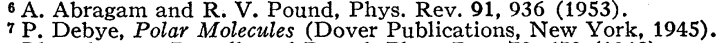

7 P. Debye, Polar Molecules (Dover Publications, New York, 1945)
8 Bioembergen, Purcell, and Pound, Phys. Rev. 73, 679 (1948).

\section{Isotopic Spin and New Unstable Particles}

$$
\begin{gathered}
\text { M. GELL-MANN } \\
\text { Department of Physics and Institute for Nuclear Studies, } \\
\text { University of Chicago, Chicago, Illinois } \\
\text { (Received August 21, 1953) }
\end{gathered}
$$

DEASLEE ${ }^{1}$ has considered the interesting possibility that the principle of charge independence, now believed to hold for nucleons and pions, may extend to the new unstable particles as well. In order to discuss this suggestion, let us suppose that both "ordinary particles" (nucleons and pions) and "new unstable particles" $\left(V_{1}, V_{4}, \tau\right.$, etc. $)$ have interactions of three kinds:

(i) Interactions that rigorously conserve isotopic spin. (We assume these to be strong.)

(ii) Electromagnetic interactions. (Let us include mass-difference effects in this category.)

(iii) Other charge-dependent interactions, which we take to be very weak.

Peaslee inquires whether the quasi stability of the $V_{1}^{0}$ may be accounted for in this way if we assume it has isotopic spin $5 / 2$. With respect to (i) the decay into pion and nucleon is absolutely forbidden. Interactions of type (iii) are supposed to be weak enough to account for the long observed lifetime of $\sim 3 \times 10^{-10}$ second. However, he concludes that effects of type (ii) will cause transitions in a very much shorter time than this, since, for example, each electromagnetic interaction can change the isotopic spin of the system by one unit.

Recently Pais ${ }^{2}$ has made the ingenious proposal that the new unstable particles differ from the ordinary ones in possessing one unit of "orbital isotopic angular momentum" and a negative "isotopic parity." If we then re-interpret (i) as referring to conservation of total isotopic angular momentum and isotopic parity, we see that as far as (i) is concerned, the decay of new unstable particles into ordinary ones is forbidden. Also, these particles will always be produced in even numbers, as Pais had suggested earlier. ${ }^{3}$ Moreover, effects of type (ii) conserve isotopic parity, as Pais has introduced it, and so do not contribute to instability of the new particles.

In connection with the work of Peaslee and of Pais, the author would like to put forward an alternative hypothesis that he has considered for some time, and which, like that of Pais, overcomes the difficulty posed by electromagnetic interactions. Let us suppose that the new unstable particles are fermions with integral isotopic spin and bosons with half-integral isotopic spin. For example, the $V_{1}$ particles may form an isotopic triplet, consisting of $V_{1}^{+}, V_{1}{ }^{0}$, and $V_{1}^{-}$. The $\tau^{+}$and $V_{4}{ }^{0}$ may form an isotopic doublet, which we may call $\tau^{+}$and $\tau^{0}$. To each of these particles there 
would presumably correspond an antiparticle, ${ }^{4}$ which we shall denote by means of square brackets.

In this scheme, (ii) is ineffective in causing decay because it can change isotopic spin only by integers, whereas in $V_{1}{ }^{\prime} \rightarrow \pi^{-}+p$, for example, the isotopic spin is 1 on the left and $\frac{1}{2}$ or $\frac{3}{2}$ on the right. Only interactions of type (iii), which do not respect isotopic spin at all, can lead to decay. Moreover, the new unstable particles again are produced only in even numbers.

There is no difficulty associated with stating a generalized Pauli principle for each kind of new unstable particle. For example, let us postulate that the wave function of a collection of $V_{1}$ 's must be totally antisymmetric in space, spin, and isotopic spin. If the wave function of two $V_{1}$ 's is antisymmetric in space and spin, as it would be for particles of identical charge, then the total isotopic spin must be 0 or 2 , which includes $V_{1}^{+} V_{1}^{+}, V_{1}^{-} V_{1}^{-}$, and $V_{1}{ }^{0} V_{1}^{0}$. If the total isotopic spin is 1 , the wave function is to be symmetric in space and spin, which is all right since the charges are then not identical. Similarly, the postulate that the wave function of a collection of $\tau$ 's must be totally symmetric in space, spin, and isotopic spin leads to no contradictions.

It should be noted that according to this scheme the conservation of the $z$ component of isotopic spin is more stringent than conservation of charge. ${ }^{5}$ To see this, let us remark that the $\tau^{+}$ and $\tau^{0}$ have $z$ components equal to $t^{\frac{1}{2}}$ and $-\frac{1}{2}$, respectively, like the proton and neutron. Correspondingly the antiparticles $\left[\tau^{+}\right]$and $\left[\tau^{0}\right]$ have $z$ components equal to $-\frac{1}{2}$ and $+\frac{1}{2}$, respectively, like the antiproton and antineutron. Thus we see that the reactions $\pi^{-}+p \rightarrow V_{1}^{0}+\tau^{0}$ and $\pi^{-}+p \rightarrow V_{1}^{-}+\tau^{+}$are allowed, while the reactions $\pi^{-}+p \rightarrow V_{1}^{0}+\left[\tau^{0}\right]$ and $\pi^{-}+p \rightarrow V_{1}^{+}+\left[\tau^{+}\right]$are forbidden, although all four are allowed by conservation of charge. In order to produce anti- $\tau$ 's it would be necessary to resort to a reaction like $\pi^{-}+p \rightarrow n+\tau^{+}+\left[\tau^{+}\right]$or $\pi^{-}+p \rightarrow n+\tau^{0}+\left[\tau^{0}\right]$.

In a similar fashion, all reactions of the form nucleon + nucleon $\rightarrow$ $V_{1}+V_{1}$ and all reactions of the form $\tau+$ nucleon $\rightarrow V_{1}+\pi$ are for bidden, while reactions such as nucleon + nucleon $\rightarrow V_{1}+\tau+$ nucleon or $[\tau]+$ nucleon $\rightarrow V_{1}+\pi$ are allowed.

1 D. C. Peaslee, Phys. Rev. 86, 127 (1952).

2 A. Pais (unpublished). The author is indebted to Professor Pais for the communication of his results prior to publication.

A. Pais, Phys. Rev. 86, 663 (1952).

We postulate the principle of invariance under the operation of charge conjugation, which carries every particle into its antiparticle. In the case of charged particles, such as the electron and the $\pi^{+}$, it is obvious that the antiparticles are the positron and the $\pi^{-}$, respectively. A neutral particle fermions, it is necessary that the neutron and the antineutron be distinct, while the question of whether the neutrino and antineutrino are distinct is one that must be settled by experiment. Among neutral bosons, the $\gamma$ ray and $\pi^{0}$ are apparently identical with their respective antiparticles, but there is no reason to believe that this is a general rule. We suppose here that the $\tau^{0}$ is a neutral boson which is not identical with its antiparticle. A mode for such a situation is provided by picturing the $\tau$ particle as a complex of a nucleon and an anti- $V_{1}$, while the $[\tau]$ is pictured as the corresponding complex of antirucleon and $V_{1}$.

5 Of course the conservation of charge is absolute, while the conservation of the $z$ component of isotopic spin can be violated by interactions of type phenomena.

\section{Differential $p-p$ Elastic-Scattering Cross Section at 144,271 , and $429 \mathrm{Mev}^{*}$}

J. Marshall, L. Marshall, and V. A. Nedzel Institute for Nuclear Studies, University of Chicago, Chicago, Illinois Received August 24, 1953)

$\mathrm{T}$

HE differential $p-p$ elastic-scattering cross section at 90 degrees barycentric angle has been determined at 144, 271, and $429 \mathrm{Mev}$ and has been found to be constant with energy within experimental error. In addition, the differential elasticscattering cross section for 429-Mev protons on liquid hydrogen has been measured as a function of angle by a scintillation counter technique which counts both incident and scattered protons, individually.

A beam of protons was scattered from a beryllium target in the 170-inch synchrocyclotron, was analyzed in the fringing field of the cyclotron magnet, and entered the experimental area through a collimator in the heavy shield. It was further monochromatized in an external magnet in the experimental area, and the proton energy was determined by range measurement. The energy of the collimated beam so obtained was varied by moving the beryllium target in the cyclotron azimuthally and at constant radius so that trajectories of the protons of desired energies would pass through the collimator. In a preliminary experiment similar to that of Oxley and co-workers, ${ }^{1}$ these beams were subjected to a second scattering on a beryllium target and analyzed for left-right scattering asymmetry. No asymmetry was found; consequently these beams are considered unpolarized for practical purposes.

The apparatus to determine the differential cross section consisted first of a pair of scintillation counters (No. 1 and No. 2), the sensitive area of each of which consisted of a one-inch square diphenyl acetylene crystal. The incident beam of monochromatic protons passed successively through these two counters and was defined by them. The beam next encountered the scatterer, a container of liquid hydrogen. The container was a Styrofoam double-walled cylinder with axis vertical and perpendicular to the beam. The incident beam passed through the two-inch Styrofoam walls, then through four inches of liquid hydrogen, and emerged through two inches of Styrofoam.

The measurements were made according to two different schemes. From $90^{\circ}$ to $54^{\circ}$ barycentric angle both elastically scattered protons were detected simultaneously and in coincidence with the incident proton. Below $54^{\circ}$ the less energetic proton begins to have too little energy to escape from the scatterer. Owing to the low intensity of the incident proton beam it was impractical to make measurements at smaller angles by decreasing the thickness of the scatterer.

Instead, a second scheme for detection of elastically scattered protons was used, relying on the fact that pion production is nearly always accompanied by a charged particle which is emitted close the the direction of the beam. When a pion is produced the associated nucleons have very little berycentric energy and, therefore, move almost with the center-of-mass velocity, consequently, almost in the direction of the beam. For example, in the reaction $p+p \rightarrow d+\pi^{+}$, the deuterons are emitted in a cone of a little less than $8^{\circ}$ half-angle around the direction of the beam. According to the first method, two liquid scintillators were placed on opposite sides of the beam at angles variable and complementary in the barycentric system. One counter (No. 4) was $9 \frac{1}{2}$ inches in diameter at a radius of 14 inches, and the other (No. 3) was $3 \frac{1}{2}$ inches in diameter at 30 inch radius and defined the solid angle of the measurement.

The first two counters were connected in double coincidence $(1,2)$ to count the number of incident protons. All four counters $(1,2,3,4)$ were connected in quadrupole coincidence to count the scattered protons. At the beam intensity which was used to measure scattered protons, the counting losses in the double coincidence were measured to be 7 percent. This correction was avoided by the use of a monitor, a 5-inch diameter liquid scintillator placed below the beam to count protons from a lead scatterer in the beam several feet past the liquid hydrogen.

The monitor was connected in double coincidence $(M, 2)$ with one of the first two counters. The counting rate in $(M, 2)$ was $\frac{1}{16}$ of the counting rate in $(1,2)$, so that when the counting losses in $(1,2)$ were as great as 7 percent the counting losses in $(M, 2)$ were negligible.

The ratio $(M, 2) /(1,2)$ was measured at low beam intensity, and the ratio $(1,2,3,4) /(M, 2)$ was measured at high beam in tensity, with and without hydrogen. The cross section is proportional to the product of the ratios with hydrogen minus the product of the ratios without hydrogen.

The background (no hydrogen) count was 14 percent of the count with hydrogen at $429 \mathrm{Mev}, 27$ percent at $271 \mathrm{Mev}$, and 12 percent at $144 \mathrm{Mev}$. The electronics were adjusted for plateaus against delays of each counter and against counter voltages. These 